\title{
COMMENT September ECI biocommentary
}

\author{
Lauren E Kelly ${ }^{1}$ \\ Pediatric Research (2018) 84:318; https://doi.org/10.1038/s41390-018-0106-9
}

I am a Pharmacologist and Certified Clinical Research Professional who grew up in Toronto, Canada. I completed an undergraduate degree in Medical Sciences at Western University, where I quickly became fascinated by how drugs worked and who they worked best for. I come from a big family and have always loved being around children. Something about the lack of existing evidence and the complex variability inspired me to pursue a career in pediatric drug research. My first research project at the Hospital for Sick Children was evaluating infant outcomes following oxycodone exposure during breastfeeding. I learned tremendously from mothers' experiences making difficult choices regarding breastfeeding and pain management in the face of very little evidence. During my PhD at Western University, I designed and conducted several clinical research projects evaluating opioid exposure in children, including a randomized clinical trial on posttonsillectomy pain management. Our trial was designed to recruit participants across three Canadian children's hospitals. An operational problem quickly emerged; all three hospitals had different pain management protocols, despite being located within $200 \mathrm{kms}$. Unable to implement our standard of care control arm, we ended up with a single-centre study.

This further sparked my interest on the rationale for clinical trial design leading me to my first post-doctoral fellowship in Clinical Research Methods back at SickKids. In this role, I had the opportunity to work with the European Medicines Agency to review Pediatric Investigation Plans. I extracted the challenges issued by regulators with regards to the proposed control arm (s) and resolutions made by the sponsor to develop a tool for justifying control arm selection in pediatric drug trials. I then sought formal training in evidence synthesis to better understand how clinical trials were appraised, combined, and compared to create practice recommendations. During my second post-doctoral fellowship at Mount Sinai Hospital, I conducted three Cochrane systematic reviews on drug safety and effectiveness in neonates.

These experiences led to my involvement with the Innovation in Pediatric Clinical Trials project, KidsCAN Trials and recruitment to the University of Manitoba as an Assistant Professor in the Department of Pediatrics and Child Health in September 2017. My advice to those coming along behind me is to develop resiliency early, as many, occasionally harsh, critiques will follow. Surround yourself with constructive, positive, and productive people. As the proverb goes, if you want to go fast, go alone. If you want to go far, go together.

Photo: Dr. Lauren E Kelly

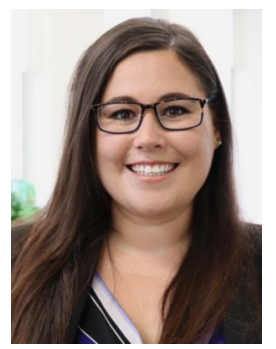

\section{ADDITIONAL INFORMATION}

Competing interests: The author declares no competing interests.

Publisher's note: Springer Nature remains neutral with regard to jurisdictional claims in published maps and institutional affiliations. 